\title{
A Novel Approach for Human Identification through Fingerprints
}

\author{
Prof G.Ramaswamy Vuda Sreenivasarao \\ Professor \\ St Mary's College \\ of Engg \& Tech \\ Hyderabad.
}

\author{
Dr.P.Ramesh D.Ravi Kiran \\ Principal Assoc.Professor \\ Donbasco Narasaraopet Engg \\ PG College College \\ Guntur Guntur
}

\begin{abstract}
Fingerprint system is an important biometric technique for personal identification. The metric used for performance of identification techniques are directly scanned fingerprints or inked impression of fingerprints. The purpose is realtime, high confidence recognition of a person's identity by mathematical analysis of the random patterns that are visible within the finger prints from some distance. Finger prints is a protected internal organ whose random texture is stable throughout life, it can serve as a kind of living password that one need not remember but one always carries along. Because the randomness of finger porint patterns has very high dimensionality, recognition decisions are made with confidence levels high enough to support rapid and reliable exhaustive searches through national-sized databases. Finger print recognition has shown to be very accurate for human identification. The metric used for performance of identification techniques are directly scanned fingerprints or inked impression of fingerprints. For better accuracy in matching the images of different decomposition of matching is performed. These parameters perform better in comparing the resultant fingerprints. When tested on a database of images this system is faster and more accurate to analyze the fingerprints matching process.
\end{abstract}

Key words: AFAS, AFIS, Image enhancement, Classification, Edge detection, Correlation and feature extraction.

\section{INTRODUCTION}

Fingerprints which have been used for about 100 years are the oldest biometrics signs of identity, scientific studies on fingerprint where initiated in the late century. But the foundations of modern fingerprint identification were established by the studies of sir F.Galton and E.Henry at the end of 19 th century. Fingerprint is formed of composite curve segments. The light areas of fingerprint are called ridges while dark areas are called valleys. The Galton's study introduced the minutiae, which are local discontinuities in the ridge patterns as discriminating features and showed the uniqueness and permanence of minutiae. According to $\mathrm{F}$ Galton fingerprint of a person is permanent i.e. it preserves its characteristics and shape from birth to death. Fingerprint of individual is unique. According to E.R.Henry, the systematic way of partitioning the fingerprint classes was so profound that it traditionally used by almost all of government security force. The names given to these classes are Right loop (R), Left loop (L), Whorl (W), Arch (A), Tented Arch (T). By using the ideas above the fingerprints, are partitioned by the Henry classification and comparing Galton features carries out extract matching. The Galton feature is details formed on ridges are defined as single curve segment. The combination of several ridges are formed by crossing and ending of the ridges are called minutiae in fingerprint literature with the increasing power of computer automated system have been developed to automate the tedious manual classification and matching method of fingerprints. There are two types first is Automatic Fingerprint Authentication System (AFAS). And second is Automatic Fingerprint Identification System (AFIS). In AFAS the input is an identity and a fingerprint image, the output is an answer of yes or no indicating whether the 
input image belongs to the person whose identity is provided. The system compares the input image with the one addressed by identity in the database. In AFIS the input is just a fingerprint and the output is a list of identities of persons that can have the given fingerprint and a score for each identity indicating the similarity between the two fingerprints. It is possible to provide partial identity information to narrow the search space. The System compares the input image with many records in the database. Recognition is defined as a process involving perception and associating the resulting information with one or combination of more than one of its memory contents. Visual perception means deriving information from a specific scene. Biometrics systems have been an important area of research in recent year. There is two important utilization of biometrics system: first is Authentication or Verification of person's identity and second is Identification in which a person's identity is sought using biometrics scene available. Any physiological or behavioral characteristics can be used to make personal identification as long as it satisfies the requirements like universality, uniqueness, performance, collect ability and Permanence.

\section{FINGERPRINT FEATURES:}

The most common representation used in fingerprint identification is the Galton features. A ridge can be defined as a single curve segment. The combination of several ridges forms a fingerprint pattern. The small features formed by crossing and ending of ridges are called minutiae. Ridge Ending \& Bifurcation are taken as the distinctive features of fingerprint. In this method the location \& angle of the feature are taken to represent the fingerprint \& used in the matching process. Together with there, fingerprint contains two special types of feature called core \& delta points. The core point is generally used as a reference point for coding minutiae \& defines as the topmost point on the innermost recurring ridge. The core $\&$ delta are also called the singularity points. Delta point is center of the triangular where 3 different directions flows meet.
Uniqueness of the Fingerprint: The "fingerprint" which is formed on the tip of the finger by the visible pattern the skin takes is absolutely unique to its owner. Every person living on the earth has a different set of fingerprints. All the people who have lived throughout history also had different fingerprints. These prints remain unchanged throughout one's lifetime unless a great injury occurs. That is why the fingerprint is accepted as a very important identity card and used for this purpose around the world.

However, two centuries ago, the fingerprint was not so important, because it was only discovered in the late 19th century that all fingerprints are different from one another. In 1880, an English scientist named Henry Faulds stated in an article published in Nature that the fingerprints of people did not change throughout their lives, and that su spects could be convicted by the fingerprints they left on surfaces such as glass. 32 In 1884 , for the first time a murder was solved by means of identifying fingerprints. Since then, fingerprints have become an important method of identification. Before the 19th century, however, people most probably had never thought that the wavy shapes on their fingertips had any meaning or considered them worthy of note. In the 7th century, the Qur'an pointed out that the fingertips of human beings bore an important characteristic.

\section{FINGERPRINT CLASSIFICATION}

Fingerprint classification is used in AFIS .The purpose of classification is clustering a database of fingerprint into as many dusters as possible. The oldest classification scheme which is used in manual fingerprint identification is the Henry classification scheme. In the scheme there are 5 classes namely arch, tented arch, right loop, left loop whorl. These classes are determined by the ridge flow on the area $\&$ the number \&relative location of core \& delta points. In this paper a novel technique is used for identification and classification. The novel technique consist the following steps: 
Step 1: Image acquisition: The aim of image acquisition subsystem can be summarized as the transformation of data into an array of numerical data which may be manipulated by a computer so the overall aim of vision may be achieved. In order to achieve this aim three major issues must be tackled and they are representation, transduction (or sensing) and digitization.

Step 2: Pre processing: The image acquired from the fingerprint sensor often results in poor quality. The poor quality image can be due to the following reasons:

1. The distortion due to elastic deformation of the finger.

2. Cuts and abrasions on the finger.

3. Dirt, oil or moist on the finger or on the scanner.

4. Partial imaging of the fingertip prints image with different rotations.

5. Non uniform contacts between finger and the sensor surface, which causes breaks in ridges and bridges

between them.

6. Contrast variation of the gray scale image.

To overcome these problems image enhancement process is necessary for accurate of the parameters. The goal of the image enhancement process is to make the non-continuous ridges and valleys of the fingerprint continuous, highly interesting foreground extraction from the noisy and irrelevant background. Median filter has been used for noise elimination.

Step 3: minutiae detection is a process of distinguish the fingerprint area in the image mostly consist of uniform background \& the fingerprint impression. In minutiae detection areas corresponding to the background are determined and discarded from the rest of the processing. The image is partitioned into blocks and each block is then labeled a background or fingerprint area. A gray level method is used for minutiae detection. In this method, we present a gray level modification of the watershed transform on grey level image. This method applies watersheds on the grey levels of neighboring pixels. For this the present paper imagines that a hole is drilled in minimum of the surface, and water will be flood from different positions into the hole. The hole is assumed to be a central pixel of a neighborhood. For the following 3X3 grey level mask the watersheds are identified as follows with a peak or highest level $\mathrm{h}>=25$. The possible watersheds of the grey level image when $h>=25$. By increasing $h$ value water will be falling from peak top points to bottom points, thus forms a good number of watersheds with different peak levels. The advantage of proposed segmentation scheme is watersheds can be easily applied on a $5 \times 5$ and also on different masks, which is not possible on a binary image.

Step 4: minutiae reduction: In minutiae reduction minutiae which are too close to each other are discarded, or minutiae which are too close to the segmented regions i.e. the region corresponding to the background or bad areas in he fingerprint, are discarded. The width of the ridges is educed to the one pixel by applying thinning technique.

Step 5: Finger print matching: Two Images are converted into binary Images and compare the images to find out the percentage of matching. In this case we need to specify some input image and that image get compared with all the other images that are present in our database. The correlation matching technique is applied for matching.

\subsection{Fingerprint recognition}

The uniqueness and permanence of the fingerprints are very well-know. Archaeological artifacts prove that fingerprints were already used by the ancient Assyrians and Chinese as a form of identification of a person. The first scientific studies on fingerprints date from the late sixteen century, but the fundamentals of modern fingerprint identification methods were provided at the end of nineteenth century. The studies of Sir F. Galton and E. Henry led to formally accept fingerprints as valid signs of identity by law enforcementagencies. The first Automated Fingerprint Identification Systems (AFIS) were developed in the 1950 s by the F.B.I. (Federal Bureau of Investigation) in cooperation with the National Bureau of Standards, the Cornell Aeronautical Laboratory and Rockwell International Corp. Ten years later other AFISs were developed by NEC Technologies Inc. (Tokyo), Printrack Inc. (Anaheim, California), and Morpho System (Paris). Fingerprint recognition is nowadays the basic 
task of the Integrated Automated Fingerprint Identification Service (IAFIS) of the most famous police agencies. Ten-print based identification and latent fingerprint recognition are the two main concerns of an IAFIS. In case of Ten-print identification, the system should identify a person by the whole sequence of his/her ten fingerprints, in case of latent figerprint the system has to identify a person through a fingerprint found on a crime scene. Technology advances in the 1980s in the areas of personal computing and optical scanners triggered non-forensic applications of fingerprint recognition methods. The enormous interest roused by electronic commerce on Internet and, more in general, by the need of reliable techniques for authenticating the identity of a living person in a broad range of applications has greatly intensified the research efforts towards the development of low cost small-size fingerprint-based biometric systems.

\subsection{Fingerprint Anatomy:}

A fingerprint is the representation of the epidermis of a finger. At a macroscopic analysis, a fingerprint is composed of a set of ridge lines which often flow parallel and sometimes produce local macro-singularities called whorl, loop and delta.
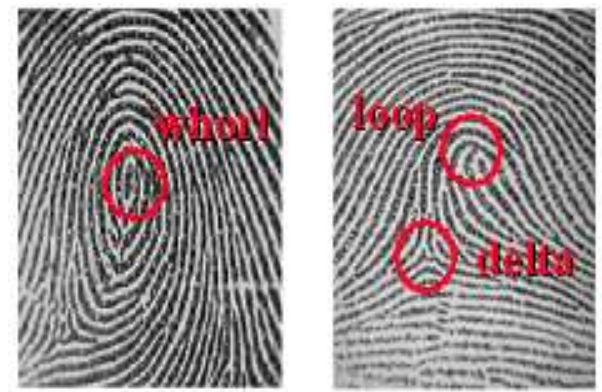

The number of cores and deltas in a single fingerprint is regulated in nature by some stringent rules; fingerprints are usually partitioned into five main classes (arch, tented arch, left loop, right loop, whorl) according to their macro-singularities.
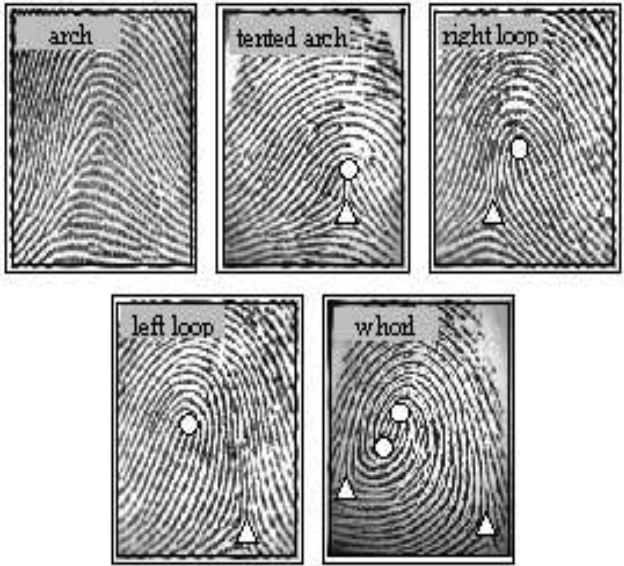

The ridge-line flow can be effectively described by a structure called directional map (or directional image) which is a discrete matrix whose elements denote the orientation of the tangent to the ridge lines. Analogously, the ridge line density can be synthesized by using a density map.

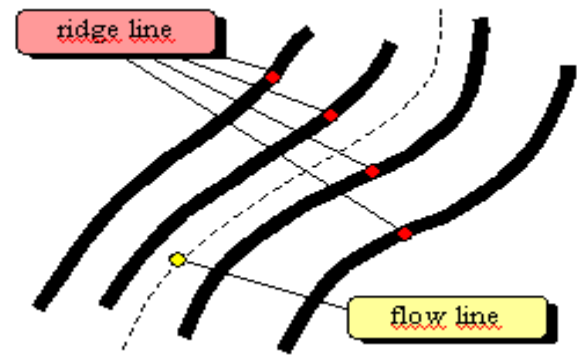

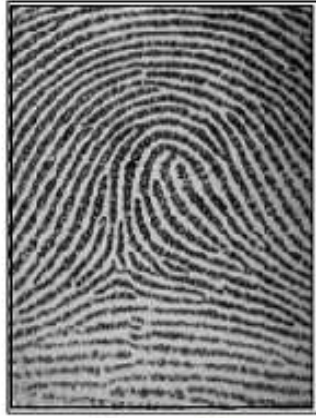

Fingerprint

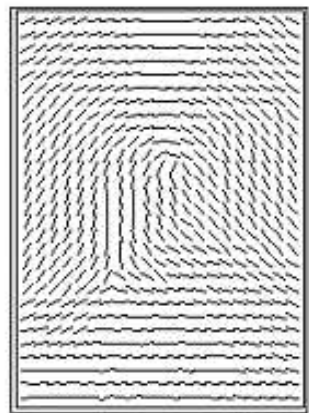

Directional image 
At a finer analysis other very important features can be discovered in the fingerprint patterns. These micro-singularities, called minutiae or Galton characteristics, are essentially determined by the termination or the bifurcation of the ridge lines. Minutiae play a primary role for fingerprint matching, since most of the algorithms rely on the coincidence of minutiae to state whether two impressions are of the same finger or not. Minutiae matching, which is essentially a point pattern matching problem, constitutes the basis of most of the automatic algorithms for fingerprint comparison.

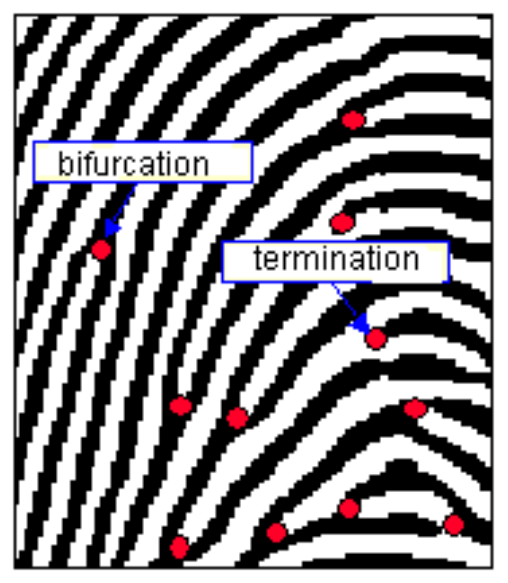

\subsection{Fingerprint acquisition:}

The oldest and most known fingerprint acquisition technique is the "ink technique", that is, pressing the finger against a card after spreading the finger skin with ink; this technique is nowadays still largely used by the police in AFIS. The cards are converted into digital form by means of scanners identical to those normally employed for general purpose paper documents. The default resolution is $500 \mathrm{dpi}$. This technique can produce images including regions which miss some information, due to excessive inkiness or to ink deficiency, and is obviously limited to forensic applications. The Frustrated Total Internal Reflection (FTIR) is the most used and mature live-scan sensing technique. The finger is illuminated from one side of a glass prism with a LED, while the other side transmits the image through a lens to a CDD/CMOS sensing element which converts light into digital information. The lack of reflection caused by the presence of water particles where the ridges touches the prism allows ridges to be discriminated from valleys.

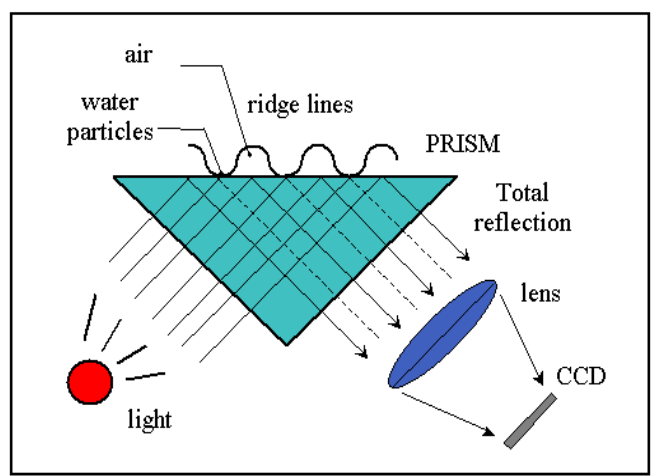

Algorithm:

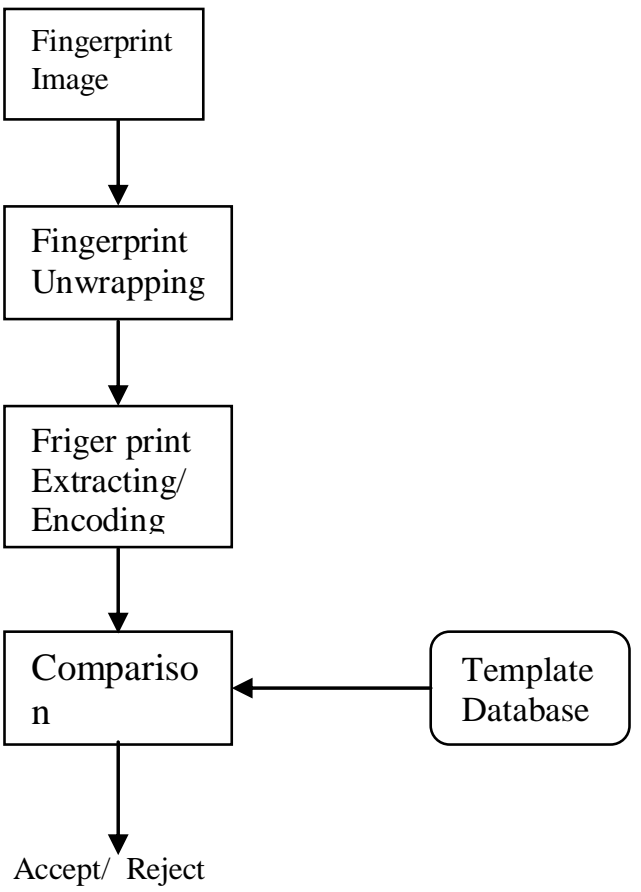

Firure: Basic design of Friger print Recognition. The algorithm were developed at Cambridge University by John Daugman. The technical performance capability of the Fingerprint recognition algorithm far surpasses that of any 
other biometric technology now available. Objective measures, such as a cross-over error rate, are at levels that cannot be reached by other biometrics. Iridian's algorithm is designed for rapid (seconds) exhaustive search of very large databases; a distinctive capability required for authentication today. The iris is an externally visible, yet protected organ whose unique epigenetic pattern remains stable throughout adult life. These characteristics make it very attractive for use as a biometric for identifying individuals. Image processing techniques can be employed to extract the unique fingerprint pattern from a digitized image of the finger, and encode it into a biometric template, which can be stored in a database. This biometric template contains an objective mathematical representation of the unique information stored in the finger, and allows comparisons to be made between templates. When a subject wishes to be identified by an fingerprint recognition system, their finger is first photographed, and then a template created for their finger region. This template is then compared with the other templates stored in a database until either a matching template is found and the subject is identified, or no match is found and the subject remains unidentified.

Fingerprint recognition allow user to hands-free operation in application. Fingerprint recognition has highest proven accuracy, had no false matches in over two million cross comparison, according to Biometric Testing Final Report. It allow high speed also for large populations. The finger is stable for each individual throughout his or her life and do not change with age.

\section{METHODOLOGY:}

Fingewrprint identification is one of the most well known and publicized bionetrics. Because of their uniqueness and consistenct over time, fingerprints have been used for Identification over century. Fingewrprint identification is popular because of the inherent ease in acquisition, the numerous sources availble for collection, and their established use and collections by law enforcement and immigration

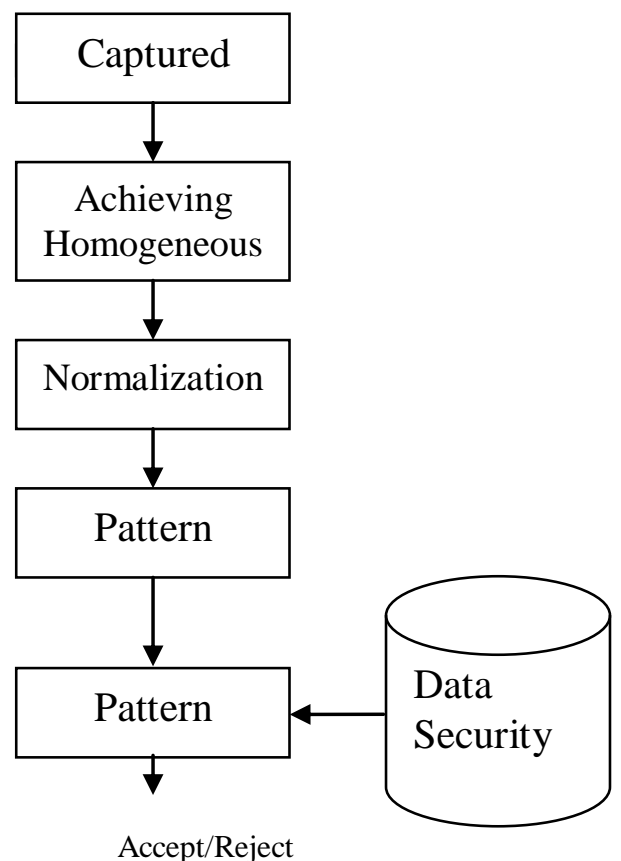

Figure: Flow chart of fringerprint Recognition.

\section{STEPS INVOLVED:}

The input is the least significant bit-plane image and the output is homogeneous masked image. We use standard deviation windows to calculate local standard deviations in the vertical and horizontal directions. By using windows, we can determine papillary boundaries. The end points in the cardinal directions $\mathrm{N}, \mathrm{E}, \mathrm{S}, \mathrm{W}$ are identified. By using these cardinal points and CIRCLE eq. we get the papillary Boundary. We get midpoints of an pupilary boundary and radius of the pupil. By calculating the distance between each pixel coordinates of image and mid points, comparing with original radius of pupil we get radius of pupil X2 + Y2 = R2

We consider $\mathrm{E}, \mathrm{W}$ points for getting the radius of the pupil and midpoint of pupil. By using Adaptive thresholding technique we can determine the limbic boundary Note that the fingerprint texture is brighter than the sclera. By finding the difference between these two regions we find out the limbic boundary value. So that we can recognize limbic boundary .we get 
midpoints of a limbic boundary and radius of the limbic. By calculating the distance between each pixel coordinates of image and mid point coordinates of limbic, comparing with original radius of limbic we get radius of limbic boundary. Fingerprint normalization and enhancement involves converting the polar coordinate system to Cartesian coordinate system. Then converting the fingerprint region from Cartesian coordinates to the normalized non-concentric polar representation is modeled as $\mathrm{I}(\mathrm{x}(\mathrm{r}, \varnothing), \mathrm{y}(\mathrm{r}, \varnothing)) \rightarrow \mathrm{I}(\mathrm{r}, \varnothing)$ With $\mathrm{x}(\mathrm{r}, \varnothing) \rightarrow(1-\mathrm{r}) \mathrm{Xp}(\varnothing)+\mathrm{rXi}(\varnothing)$ $\mathrm{y}(\mathrm{r}, \varnothing) \rightarrow(1-\mathrm{r}) \mathrm{Yp}(\varnothing)+\mathrm{rYi}(\varnothing)$ where $\mathrm{I}(\mathrm{x}, \mathrm{y})$ is the fingerprint region images $(\mathrm{x}, \mathrm{y})$ are the orginal Cartesian coordinates $(\mathrm{r}, \varnothing)$ are the corresponding normalized polar coordinates and $\mathrm{Xp}, \mathrm{Yp}$ and $\mathrm{Xi}, \mathrm{Yi}$ are the coordinates of pupil and iris boundary along o direction .

Note : $\varnothing$ varies from 0 t0 $360 \mathrm{r}$ varies from 0 to $\mathrm{Ri}-\mathrm{Rp}$ where $\mathrm{Ri}=$ Radius of fingerprint, $\mathrm{Rp}=$ radius of pupil Converting enhanced image into logical image

Pattern Recognition: Texture near the papillary boundary and limbic boundary inside the iris has some errors. So we take the middle row of iris in order to overcome the errors. We convert the middle row of bits in to hexadecimal code. In our project the probability of occurring secret code is nearly $16^{90}$. We consider 360 bits of middle row of an enhanced image. Convert these bits into hexadecimal code.

$\mathrm{F}=100111101100 \ldots 01101100010111011011$

$$
\begin{array}{llllllll}
9 & \mathrm{E} & \mathrm{C} & 6 & \mathrm{C} & 5 & \mathrm{D} & \mathrm{B}
\end{array}
$$

(360 Bits code)-----(90 Hexadecimal code)

Pattern Matching: Hexadecimal code is taken from database and converted into bits. The comparison is done by computing the HAMMING DISTANCE between the two codes. The Hamming distance between an Iris code A and another code B is given by

$$
H D=\frac{1}{N} \sum_{j=1}^{N} A_{j} \otimes B_{j}
$$

Hamming Distance: Given two patterns A and B the sum of disagreeing bits (sum of the exclusive-OR between) divided by $\mathrm{N}$ the total number of bits in the pattern. If two patterns are derived from the same iris, the hamming distance between them will be close to 0.0 then it is accepted or else rejected.

\section{RESULTS:}

Fingerprint is recognized by using simple methods and the less complex and faster algorithms than previous algorithms and it eliminates image noises and reflections. Homogenization removes specularities of the images. Bit

plane slicing, morphological operations and standard deviations windows helps to recognize image radius and image mid-point. By solving these parameters in circle equation, we can recognize pupilary boundary (inner boundary) accurately. Adaptive threshoulded method can find the limbic radius and limbic mid-point. By solving these parameters in circle equation, we can recognize limbic boundary (outer boundary) accurately. The region between inner and outer boundary is fingerprint, it is in the polar form and converted into linear form by converting the polar coordinate system to Cartesian coordinate system, then converting the finger region from Cartesian coordinates to the normalized nonconcentric polar representation we get normalized image. By doing enhancement, the logical image with 360 in length and breadth is the difference between the outer and inner boundary is produced. The texture near the limbic and pupilary boundary inside the iris has some noises due to eyelashes and eyelids, by taking the middle row of the enhanced image a secret code is extracted from it. The secret code is converted into Hexadecimal code of length 90. Hamming code distance is being used for pattern matching. It can give the 1690 different fingerprint codes. It can overcome the noises caused by pupil in the image

\section{REFERENCES}

[1] Digital Image Processing by Fafael C.Gonzalez,Richard E.Woods Addison westly pub.company.

[2] Fundamentals of digital image processing by A.K.Jain.

[3] Introduction to finger print Recognition by U. Halici, Turkey,L.C. Jain,Australia \& A.Erol, Turkey. 
[4] Finger print feature processing technique \& poroscope by A.R.Reddy\& J.D. Stosz,U.S.A.

[5] A gaber filter-based method for finger print Identification by Y.Hamamoto,japan.

[6] WWW.GOOGLE.COM

[7] WWW.MATHWORKS.COM

[8] WWW.DOWNLOADS.COM

[9] R.C.Gonzalez, Richard E. Woods, Digital Image Processing, Pearson education.

\section{AUTHOR'S BIOGRAPHY}

Prof.G.Ramaswamy received the M.Tech degree in Information Technology from the Punjabi University, in 2003. He received the Ph.D degree in Computer Science \& Engg from Magadh University from the 2007. Currently he is professor in St.Mary's College of Engg \& Tech. His research interests include Network Security, Cryptography \& Image Processing.
Vuda.Sreenivasarao received the M.Tech degree in Computer Science\& Engg from the Satyabama University, in 2007. . He is research student of Annamalia University. Currently he is Assoc.Professor in St.Mary's College of Engg \& Tech. His research interests include Network Security, Cryptography and Data mining.

Dr. P.Ramesh Babu working as Principal, Don Bosco P.G. College, Guntur. He has completed his M.Sc ( $\mathrm{C} \mathrm{S}$ ) From Bharathi Dasan University. He got M.Tech (I.T ) From Punjabi University. He received Ph.D (C.S.E) From Magadh University. His research interests include Network Security, Cryptography and Artificial Intellegence.

Mr. D.Ravi Kiran received the M.C.A. degree from Bhavnagar University, Gujarath in 1999 and M.Tech Information Technology from Punjabi University, Patiala in 2004. Currently working as a faculty member in Narasaraopet Engineering College, Narasaraopet. His areas of interest are in Network Security, Biometrics and Image Processing. 